\title{
Construction of Solitary Wave Solutions and Rational Solutions for mKdV Equation with Initial Value Problem by Homotopy Perturbation Method
}

\author{
Zhongzhou Dong, Fen Wang \\ School of Mathematics and Information Science, Henan Polytechnic University, Jiaozuo, China \\ Email: zzdong@hpu.edu.cn
}

How to cite this paper: Dong, Z.Z. and Wang, F. (2018) Construction of Solitary Wave Solutions and Rational Solutions for mKdV Equation with Initial Value Problem by Homotopy Perturbation Method. Open Access Library Journal, 5: e4383. https://doi.org/10.4236/oalib.1104383

Received: January 27, 2018

Accepted: February 25, 2018

Published: February 28, 2018

Copyright ( 2018 by authors and Open Access Library Inc.

This work is licensed under the Creative Commons Attribution International License (CC BY 4.0).

http://creativecommons.org/licenses/by/4.0/

\section{(c) (i) Open Access}

\begin{abstract}
The $\mathrm{mKdV}$ equation with the initial value problem is studied numerically by means of the homotopy perturbation method. The analytical approximate solutions of the $\mathrm{mKdV}$ equation are obtained. Choosing the form of the initial value, the single solitary wave, two solitary waves and rational solutions are presented, some of which are shown by the plots.
\end{abstract}

\section{Subject Areas \\ Partial Differential Equation}

\section{Keywords}

mKdV Equation, Homotopy Perturbation Method, Soliton Solution

\section{Introduction}

Partial differential equations widely describe many phenomena in the world. Although many mathematicians and physicists presented various methods to find the explicit solutions of the partial differential equations, it is a difficult and important task to build the solutions of initial and boundary value problem. Recently, the homotopy perturbation method (HPM) have been applied into many problems [1]-[10] and tested to be an effective tool. Here, the initial value problem of the $\mathrm{mKdV}$ equation is studied by using HPM.

The initial value problem of $\mathrm{mKdV}$ equation is as following:

$$
\left\{\begin{array}{l}
u_{t}+6 u^{2} u_{x}+u_{x x x}=0 \\
u(x, 0)=f(x)
\end{array}\right.
$$


The mKdV equation arises in many different fields, such as shallow water model, plasma science, biophysics and so on. A Darboux transformation was developed for generating dark multi-soliton solutions of the mKdV equation [11]. Based on the factorization of soliton equations into two commuting integrable $\mathrm{x}$ - and $\mathrm{t}$-constrained flows, Ref. [12] derived $\mathrm{N}$-soliton solutions for mKdV equation via its $\mathrm{x}$ - and t-constrained flows. Ref. [13] obtained numerical and exact compacton solutions of Equation (1) using by the variational iteration method. By using the extended tanh method, Wazwaz [14] got the abundant solitary wave solutions of the mKdV equation. Wazwaz [15] introduced new schemes to study the solitary wave solutions of the mKdV equation. Ref. [16] obtained the exact periodic solitary-wave solutions of the $\mathrm{mKdV}$ equation by the extended homoclinic test method. Applying the nonlocal conservation theorem method and the partial Lagrangian approach to the $\mathrm{mKdV}$ equation, the conservation laws were presented in Ref. [17]. From the observations on the tanh-coth expansion method, Parkes [18] found new solutions of the mKdV equation. By the bilinear approach, Ref. [19] obtained a symmetry constraint system and $\mathrm{N}$-soliton solutions as group invariant solutions for the $\mathrm{mKdV}$ equation. In Ref. [20], ehe authors obtained an efficient numerical method to study the asymptotic solution of Equation (1). The authors studied compact solitary waves of the mKdV equation by using the phase portrait theory [21]. From the known Lax pair, Ref. [22] studied the nonlocal symmetry, optimal systems, and explicit solutions of the $\mathrm{mKdV}$ equation.

This paper is arranged as follows: In Section 2, by using HPM, we obtain the analytical approximate solution of Equation (1). In Section 3, by taking the form of the initial value, some exact solutions of $\mathrm{mKdV}$ equation are obtained. And some pictures are given to show the structure of the obtained solutions. Finally, some conclusions and discussions are given in Section 4.

\section{The Homotopy Perturbation Method to mKdV Equation}

In order to obtain the analytical approximate solution of Equation (1), we consider the one-parameter family of Equation (1) as follows

$$
\left(u-u_{0}\right)_{t}+p\left(6 u^{2} u_{x}+u_{x x x}\right)=0
$$

where the parameter $p \in[0,1]$ and $u_{0}=f(x)$.

If $p=0$, we meet $u=u_{0}$.

If $p=1$, we come back to the original problem (1). Let the solution $u(x, t)$ of the system (2) be written in the form of an infinite series,

$$
u(x, t)=\sum_{i=0}^{\infty} u_{i}(x, t) p^{i}
$$

Then $u(x, t)=\sum_{i=0}^{\infty} u_{i}(x, t)$ is a series solution of Equation (1).

Substituting Equation (3) into Equation (2), and equating the coefficients of $p, p^{2}, \cdots$, we have

$$
u_{1, t}+6 u_{0}^{2} u_{0, x}+u_{0, x x x}=0
$$




$$
\begin{gathered}
u_{2, t}+6 u_{0}^{2} u_{1, x}+u_{1, x x x}+12 u_{0} u_{1} u_{0, x}=0, \\
u_{3, t}+6 u_{0}^{2} u_{2, x}+6 u_{1}^{2} u_{0, x}+u_{2, x x x}+12 u_{0} u_{1} u_{1, x}+12 u_{0} u_{2} u_{0, x}=0,
\end{gathered}
$$

and so on. Solving Equations (4), (5) and (6), one can obtain

$$
\begin{aligned}
& u_{1}(x, t)=-\left(6 u_{0}^{2} u_{0, x}+u_{0, x x x}\right) t, \\
& u_{2}(x, t)=\frac{1}{2}\left(144 u_{0}^{3} u_{0, x}^{2}+36 u_{0}^{4} u_{0, x x}+12 u_{0}^{2} u_{0, x x x x}+72 u_{0, x}^{2} u_{0, x x}\right. \\
& \left.+36 u_{0} u_{0, x x}^{2}+60 u_{0} u_{0, x} u_{0, x x x}+u_{0, x x x x x x}\right) t^{2}, \\
& u_{3}(x, t)=-\frac{1}{6}\left(900 u_{0, x} u_{0, x x x}^{2}+6480 u_{0}^{4} u_{0, x}^{3}+864 u_{0, x}^{5}+504 u_{0} u_{0, x x x} u_{0, x x x x}\right. \\
& +2376 u_{0}^{3} u_{0, x x} u_{0, x x x}+6264 u_{0}^{2} u_{0, x}^{2} u_{0, x x x}+1512 u_{0}^{3} u_{0, x} u_{0, x x x x} \\
& +3888 u_{0}^{5} u_{0, x} u_{0, x x}+7992 u_{0}^{2} u_{0, x} u_{0, x x}^{2}+144 u_{0} u_{0, x} u_{0, x x x x x x}+324 u_{0} u_{0, x x} u_{0, x x x x x} \\
& +1404 u_{0, x} u_{0, x x} u_{0, x x x x}+324 u_{0, x}^{2} u_{0, x x x x x}+108 u_{0}^{4} u_{0, x x x x x}+216 u_{0}^{6} u_{0, x x x} \\
& \left.+1296 u_{0, x x}^{2} u_{0, x x x}+18 u_{0}^{2} u_{0, x x x x x x x}+9504 u_{0} u_{0, x}^{3} u_{0, x x}+u_{0, x x x x x x x x x}\right) t^{3} .
\end{aligned}
$$

Hence, we obtain the solution of Equation (1)

$$
u(x, t)=f(x)+u_{1}(x, t)+u_{2}(x, t)+u_{3}(x, t)+\cdots,
$$

where $u_{1}(x, t), u_{2}(x, t)$ and $u_{3}(x, t)$ are given by Equations (7), (8) and (9) respectively.

\section{Application}

In this section, we will study the single soliton, two-soliton and rational solutions of $\mathrm{mKdV}$ equation.

\subsection{Single Solitary Wave Solution}

Consider the following case:

$$
\left\{\begin{array}{l}
u_{t}+6 u^{2} u_{x}+u_{x x x}=0 \\
u(x, 0)=-\frac{2 k \exp (k x)}{\exp (2 k x)+1} .
\end{array}\right.
$$

From the above section, we can have

$$
\begin{gathered}
u_{0}(x, t)=-\frac{2 k \exp (k x)}{\exp (2 k x)+1}, \\
u_{1}(x, t)=-\frac{2 k^{4} \exp (k x)(\exp (2 k x)-1) t}{(\exp (2 k x)+1)^{2}}, \\
u_{2}(x, t)=-\frac{k^{7} \exp (k x)(\exp (4 k x)-6 \exp (2 k x)+1) t^{2}}{(\exp (2 k x)+1)^{3}}, \\
u_{3}(x, t)=-\frac{k^{10} \exp (k x)(\exp (6 k x)-23 \exp (4 k x)+23 \exp (2 k x)-1) t^{3}}{3(\exp (2 k x)+1)^{4}},
\end{gathered}
$$




$$
\begin{aligned}
u(x, t)= & -\frac{2 k \exp (k x)}{\exp (2 k x)+1}-\frac{2 k^{4} \exp (k x)(\exp (2 k x)-1)}{(\exp (2 k x)+1)^{2}} t \\
& -\frac{k^{7} \exp (k x)(\exp (4 k x)-6 \exp (2 k x)+1)}{(\exp (2 k x)+1)^{3}} t^{2} \\
& -\frac{k^{10} \exp (k x)(\exp (6 k x)-23 \exp (4 k x)+23 \exp (2 k x)-1)}{3(\exp (2 k x)+1)^{4}} t^{3}+\cdots
\end{aligned}
$$

Using Taylor series, one can obtain the exact solution

$$
u(x, t)=-\frac{2 k \exp \left(k\left(x-k^{2} t\right)\right)}{\exp \left(2 k\left(x-k^{2} t\right)\right)+1} .
$$

Figure 1 shows the single soliton (10) for $k=-1,-4 \leq x \leq 4$ and $-4 \leq t \leq 4$. Figure 2 shows the single soliton (10) for $k=-1,-4 \leq x \leq 4$ and $t=0$.

\subsection{Two Solitary Waves Solution}

In this case, we take

$$
f(x)=\frac{4 \exp (x)}{\exp (2 x)+1}
$$

Then from the above section, one can have

$$
u_{0}(x, t)=\frac{4 \exp (x)}{\exp (2 x)+1}
$$

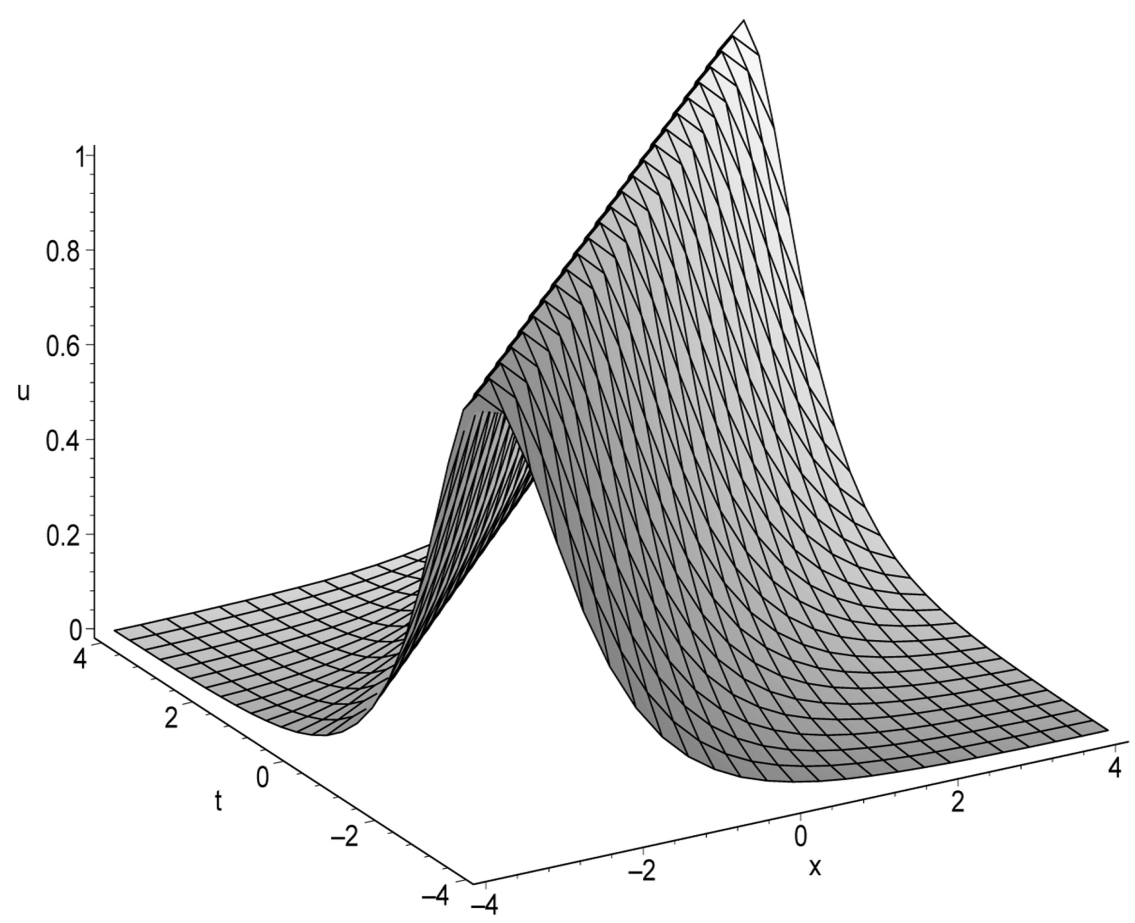

Figure 1.3D plot of solution (10) for $k=-1$. 


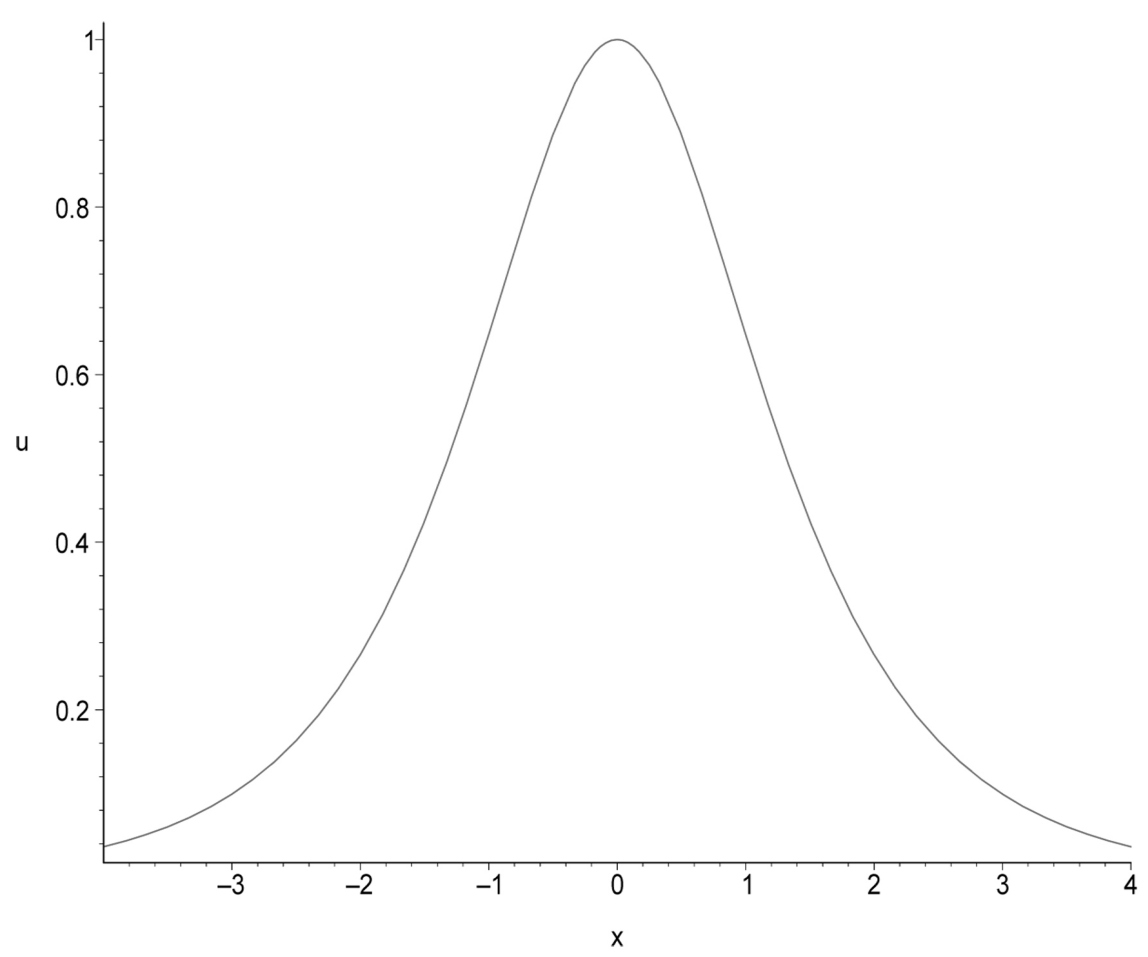

Figure 2. Plot of solution (10) for $k=-1$ and $t=0$.

$$
\begin{aligned}
& u_{1}(x, t)=\frac{4 \exp (x)}{(\exp (2 x)+1)^{4}}(\exp (6 x)+73 \exp (4 x)-73 \exp (2 x)-1) t \\
& u_{2}(x, t)=\frac{2 \exp (x)}{(\exp (2 x)+1)^{7}}(\exp (12 x)+2158 \exp (10 x)+2863 \exp (8 x) \\
& -26236 \exp (6 x)+2863 \exp (4 x)+2158 \exp (2 x)+1) t^{2} \text {, } \\
& u_{3}(x, t)=\frac{2 \exp (k x)}{3(\exp (2 x)+1)^{10}}(\exp (18 x)+58951 \exp (16 x)+225620 \exp (14 x) \\
& -1999268 \exp (12 x)-6147250 \exp (10 x)+6147250 \exp (8 x) \\
& +1999268 \exp (6 x)-225620 \exp (4 x)-58951 \exp (2 x)-1) t^{3}, \\
& u(x, t)=\frac{4 \exp (x)}{\exp (2 x)+1}+\frac{4 \exp (x)}{(\exp (2 x)+1)^{4}}(\exp (6 x)+73 \exp (4 x)-73 \exp (2 x)-1) t \\
& +\frac{2 \exp (x)}{(\exp (2 x)+1)^{7}}(\exp (12 x)+2158 \exp (10 x)+2863 \exp (8 x) \\
& -26236 \exp (6 x)+2863 \exp (4 x)+2158 \exp (2 x)+1) t^{2} \\
& +\frac{2 \exp (k x)}{3(\exp (2 x)+1)^{10}}(\exp (18 x)+58951 \exp (16 x)+225620 \exp (14 x) \\
& -1999268 \exp (12 x)-6147250 \exp (10 x)+6147250 \exp (8 x) \\
& +1999268 \exp (6 x)-225620 \exp (4 x)-58951 \exp (2 x)-1) t^{3}+\cdots \text {. }
\end{aligned}
$$

Using Taylor series, one can obtain the exact solution 


$$
u(x, t)=\frac{4(\exp (t-x)+3 \exp (27 t-3 x)+3 \exp (29 t-5 x)+\exp (55 t-7 x))}{1+4 \exp (2 t-2 x)+6 \exp (28 t-4 x)+4 \exp (54 t-6 x)+\exp (56 t-8)}
$$

Figure 3 shows the two-soliton solution (11) for $-5 \leq x \leq 5$ and $-0.5 \leq t \leq 0.5$. Figure 4 shows the two-soliton solution (11) for $-6 \leq x \leq 6$ and $t=-0.2$. Figure 5 shows the two-soliton solution (11) for $-4 \leq x \leq 4$ and $t=0$. Figure 6 shows the two-soliton solution (11) for $-6 \leq x \leq 6$ and $t=0.2$.

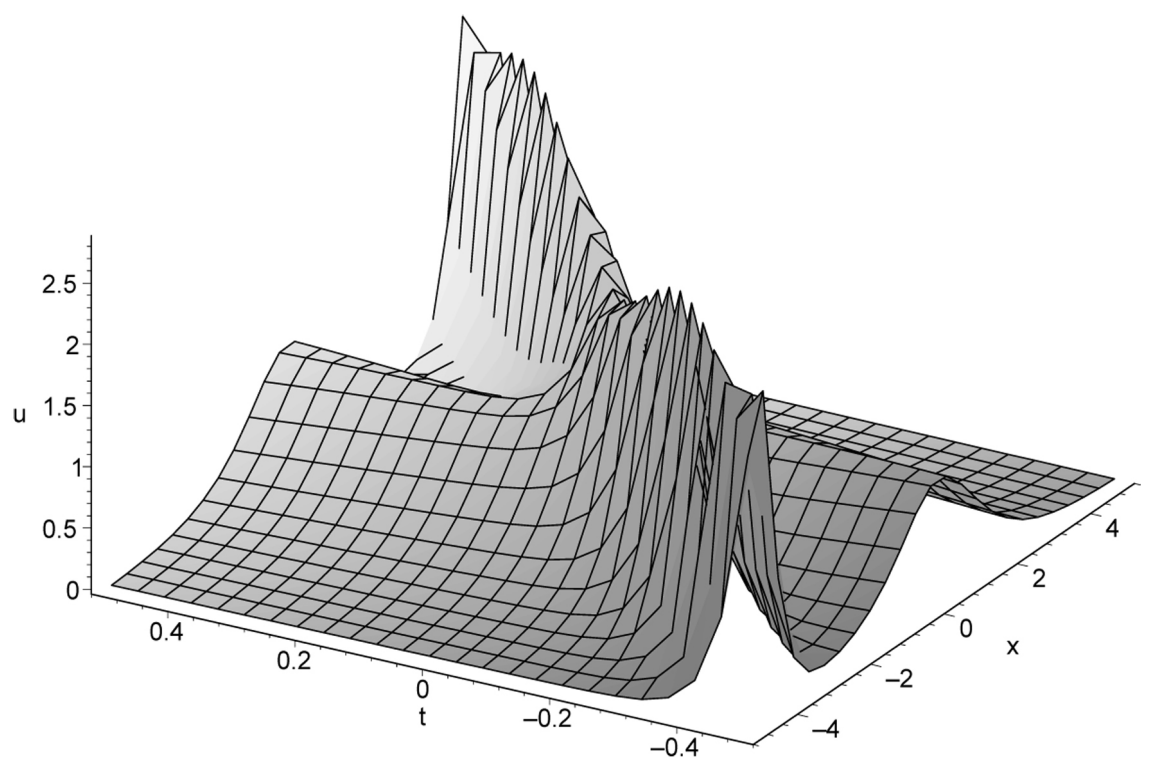

Figure 3. 3D plot of solution (11).

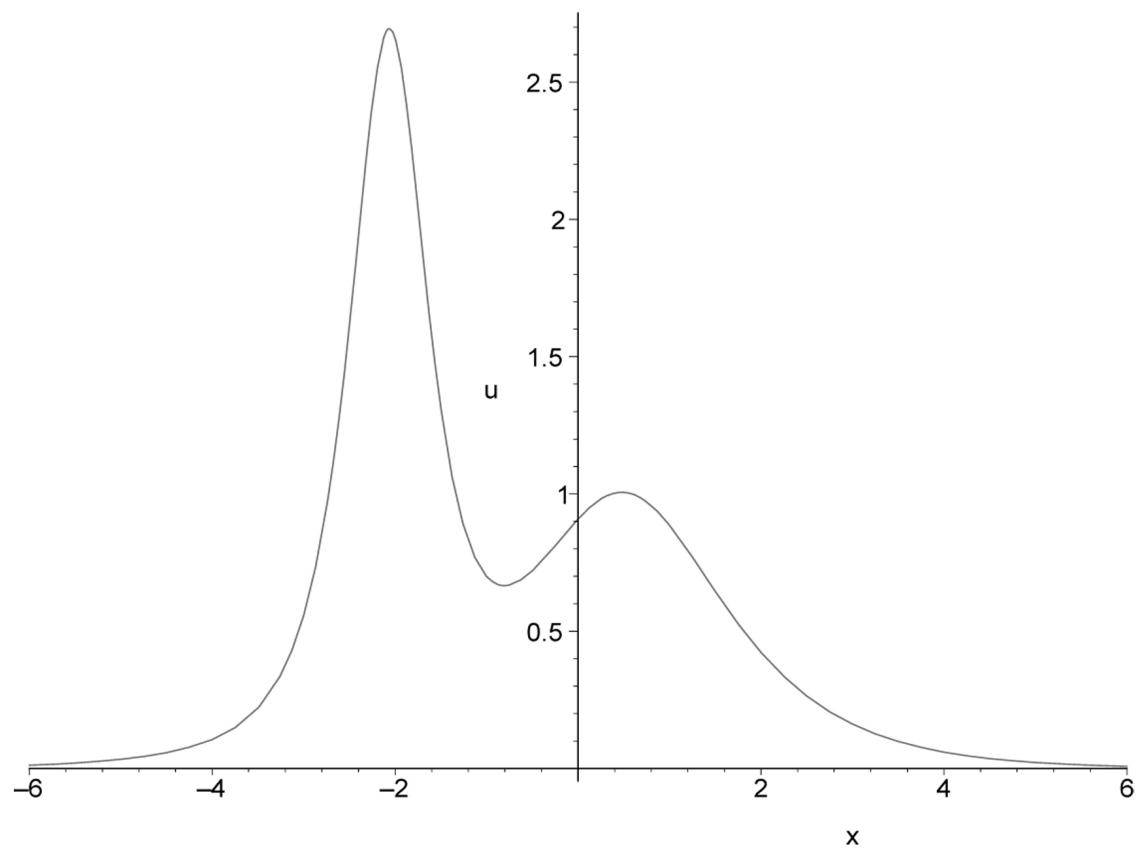

Figure 4. Plot of solution (11) for $t=-0.2$. 


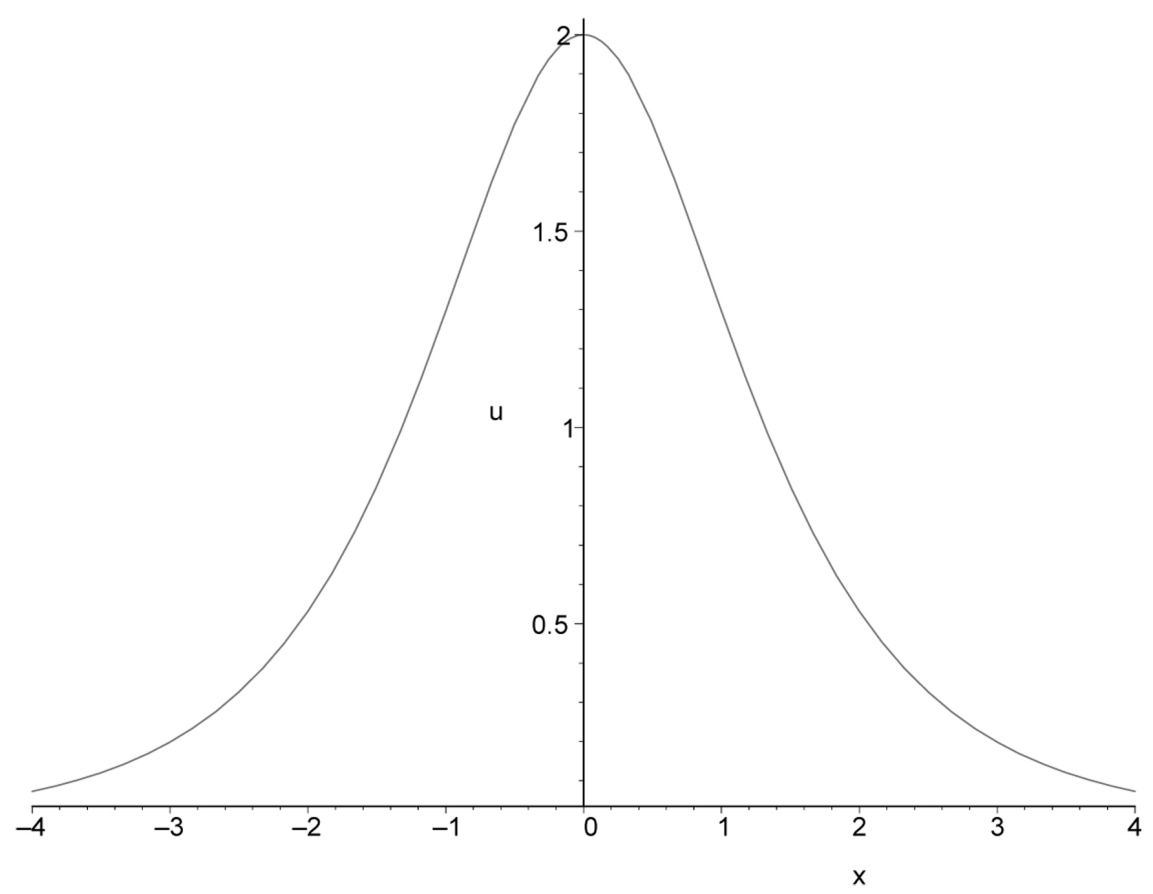

Figure 5. Plot of solution (11) for $t=0$.

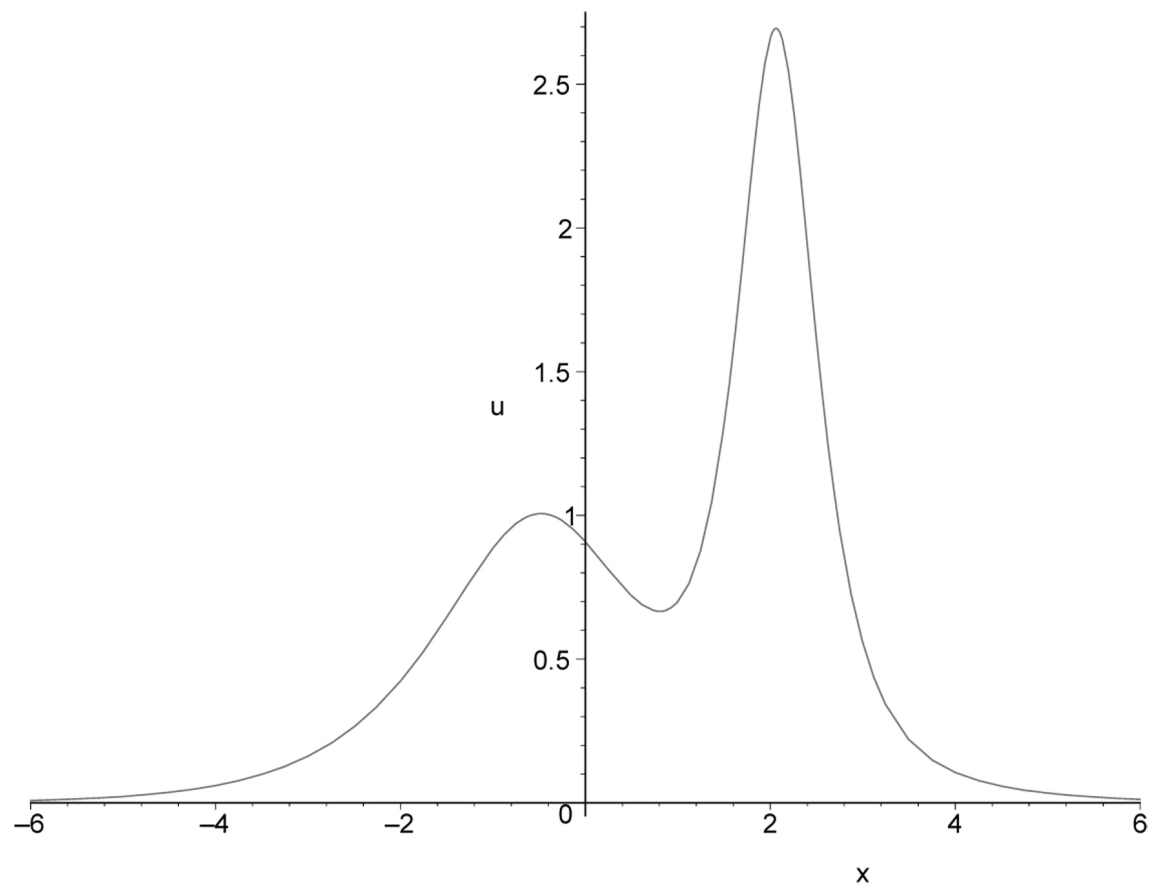

Figure 6. Plot of solution (11) for $t=0.2$.

\subsection{Rational Solution}

Here, our goal is to find the rational solution of mKdV equation. To do this, we consider the form of the initial value as follows:

$$
f(x)=\frac{2 I}{x-a}
$$


Due to the above section, it is obtained

$$
\begin{gathered}
u_{0}(x, t)=\frac{2 I}{x-a}, \\
u_{1}(x, t)=-\frac{36 I t}{(x-a)^{4}}, \\
u_{2}(x, t)=\frac{432 I t^{2}}{(x-a)^{7}}, \\
u(x, t)=\frac{2 I}{x-a}-\frac{36 I}{(x-a)^{4}} t+\frac{432 I}{(x-a)^{7}} t^{2}-\frac{5184 I}{(x-a)^{10}} t^{3}+\cdots .
\end{gathered}
$$

From the knowledge of Taylor series, one can get the exact solution

$$
u(x, t)=\frac{2 I\left[(x-a)^{3}-6 t\right]}{(x-a)\left[(x-a)^{3}+12 t\right]},
$$

which is singular at $x=a$ or $(x-a)^{3}+12 t=0$.

\section{Conclusion}

In summary, we successfully apply homotopy perturbation method to the $\mathrm{mKdV}$ equation with the initial value problem and obtain the analytical approximate solution of the $\mathrm{mKdV}$ equation. Using the form of the initial value, the single solitary wave, two solitary waves and rational solutions of the $\mathrm{mKdV}$ are obtained. Here, we get the two solitary waves solution without using bilinear forms, Wronskian, etc. In our later works, we will focus on the form of the initial value that can create the two solitary waves solutions.

\section{Acknowledgements}

This work was supported by the National Natural Science Foundation of China under Grant No. 11305048, the Science and Technology Research Key Project of Education Department of Henan Province under Grant No. 13A110329, the Basic and Frontier Research Program of Henan Province under Grant No. 132300410223, the Doctor Foundation of Henan Polytechnic University under Grant No. B2011-006, and the Key Teacher Foundation of Henan Polytechnic University (Grant 2014).

\section{References}

[1] Chow, S.N., Mallet-Paret, J. and Yorke, J.A. (1978) Finding Zeros of Maps: Homotopy Methods That Are Constructive with Probability One. Mathematics of Computation, 32, 887-899. https://doi.org/10.1090/S0025-5718-1978-0492046-9

[2] Watson, L.T. (1986) Numerical Linear Algebra Aspects of Globally Convergent Homotopy Methods. SIAM Review, 28, 529-545. https://doi.org/10.1137/1028157 
[3] Li, T.Y. and Sauser, T. (1987) Homotopy Methods for Eigenvalue Problems. Linear Algebra and Its Applications, 91, 65-74. https://doi.org/10.1016/0024-3795(87)90060-7

[4] Liao, S.J. (1995) An Approximate Solution Technique Not Depending on Small Parameters: A Special Example. International Journal of Non-Linear Mechanics, 30, 371-380. https://doi.org/10.1016/0020-7462(94)00054-E

[5] He, J.H. (2004) Comparison of Homotopy Perturbation Method and Homotopy Analysis Method. Applied Mathematics and Computation, 156, 527-539. https://doi.org/10.1016/j.amc.2003.08.008

[6] Ganji, D.D. and Sadighi, A. (2006) Application of He's Homotopy-Perturbation Method to Nonlinear Coupled Systems of Reaction-Diffusion Equations. International Journal of Nonlinear Sciences and Numerical Simulation, 7, 411-418. https://doi.org/10.1515/IJNSNS.2006.7.4.411

[7] Javidi, M. and Golbabai, A. (2007) A Numerical Solution for Solving System of Fredholm Integral Equations by Using Homotopy Perturbation Method. Applied Mathematics and Computation, 189, 1921-1928.

https://doi.org/10.1016/j.amc.2006.12.070

[8] Wang, Q. (2007) Homotopy Perturbation Method for Fractional KdV Equation. Applied Mathematics and Computation, 190, 1795-1802. https://doi.org/10.1016/j.amc.2007.02.065

[9] Odibat, Z. (2007) A New Modification of the Homotopy Perturbation Method for Linear and Nonlinear Operators. Applied Mathematics and Computation, 189, 746-753. https://doi.org/10.1016/j.amc.2006.11.188

[10] Abbasbandy, S. (2007) Application of He's Homotopy Perturbation Method to Functional Integral Equations. Chaos Solitons \& Fractals, 31, 1243-1247. https://doi.org/10.1016/j.chaos.2005.10.069

[11] Chen, Z.Y., Huang, N.N., Liu, Z.Z. and Xiao, Y. (1993) An Explicit Expression of the Dark $N$-Soliton Solution of the MKdV Equation by Means of the Darboux Transformation. Journal of Physics A: Mathematical and General, 26, 1365-1374. https://doi.org/10.1088/0305-4470/26/6/018

[12] Zeng, Y.B. and Dai, H.H. (2001) Constructing the $N$-Soliton Solution for the $\mathrm{mKdV}$ Equation through Constrained Flows. Journal of Physics A: Mathematical and General, 34, L657-L663. https://doi.org/10.1088/0305-4470/34/46/103

[13] Inc, M. (2007) An Approximate Solitary Wave Solution with Compact Support for the Modified KdV Equation. Journal of Applied Mathematics and Computing, 184, 631-637. https://doi.org/10.1016/j.amc.2006.06.062

[14] Wazwaz, A.M. (2007) The Extended Tanh Method for Abundant Solitary Wave Solutions of Nonlinear Wave Equations. Journal of Applied Mathematics and Computing, 187, 1131-1142. https://doi.org/10.1016/j.amc.2006.09.013

[15] Wazwaz, A.M. (2008) New Sets of Solitary Wave Solutions to the KdV, mKdV, and the Generalized KdV Equations. Communications in Nonlinear Science and $\mathrm{Nu}$ merical Simulation, 13, 331-339. https://doi.org/10.1016/j.cnsns.2006.03.013

[16] Gao, Y.F., Dai, Z.D. and Li, D. (2009) New Exact Periodic Solitary-Wave Solution of $\mathrm{mKdV}$ Equation. Communications in Nonlinear Science and Numerical Simulation, 14, 3821-3824. https://doi.org/10.1016/j.cnsns.2008.09.011

[17] Yasar, E. (2010) On the Conservation Laws and Invariant Solutions of the mKdV Equation. Journal of Mathematical Analysis and Applications, 363, 174-181. https://doi.org/10.1016/j.jmaa.2009.08.030 
[18] Parkes, E.J. (2010) Observations on the Tanh-Coth Expansion Method for Finding Solutions to Nonlinear Evolution Equations. Journal of Applied Mathematics and Computing, 217, 1749-1754. https://doi.org/10.1016/j.amc.2009.11.037

[19] Zhang, J.B., Zhang, D.J. and Shen, Q. (2011) Bilinear Approach for a Symmetry Constraint of the Modified KdV Equation. Journal of Applied Mathematics and Computing, 218, 4494-4500. https://doi.org/10.1016/j.amc.2011.10.030

[20] Trogdon, T., Olver, S. and Deconinck, B. (2012) Numerical Inverse Scattering for the Korteweg-de Vries and Modified Korteweg-de Vries Equations. Physica D, 241, 1003-1025. https://doi.org/10.1016/j.physd.2012.02.016

[21] Yin, J.L., Xing, Q.Q. and Tian, L.X. (2014) Complex Dynamical Behaviors of Compact Solitary Waves in the Perturbed mKdV Equation. Chinese Physics B, 23, 080201. https://doi.org/10.1088/1674-1056/23/8/080201

[22] Xin, X.P., Miao, Q. and Chen, Y. (2014) Nonlocal Symmetry, Optimal Systems, and Explicit Solutions of the mKdV Equation. Chinese Physics B, 23, 010203. https://doi.org/10.1088/1674-1056/23/1/010203 